PROCEEDINGS OF THE

AMERICAN MATHEMATICAL SOCIETY

Volume 139, Number 8, August 2011, Pages 2839-2846

S 0002-9939(2011)10718-4

Article electronically published on January 6, 2011

\title{
HYPERCYCLIC WEIGHTED TRANSLATIONS ON GROUPS
}

\author{
C. CHEN AND C-H. CHU
}

(Communicated by Nigel J. Kalton)

\begin{abstract}
Let $G$ be a locally compact group and let $1 \leq p<\infty$. We characterize hypercyclic weighted translation operators on the Lebesgue space $L^{p}(G)$ in terms of the weight, extending a recent result by the authors for discrete groups. Topologically mixing weighted translations are also characterized.
\end{abstract}

\section{INTRODUCTION}

Recently, we characterized in [6] hypercyclic weighted translations by aperiodic elements on coset spaces of discrete groups. In this paper, we complete our investigation by extending the characterization to all non-discrete coset spaces. In fact, we adopt an approach different from that in [6], where the Hypercyclic Criterion of Kitai [15, Gethner and Shapiro [10] was used. The proof in this paper does not depend on this criterion but makes use of a weaker condition of topological transitivity, and unifies both the discrete and non-discrete cases. This fresh approach entails two further consequences, one is the equivalence of hypercyclicity and the weakly mixing condition for weighted translations, the other is a characterization of topologically mixing weighted translations on coset spaces.

We recall that a continuous linear self-map $T$ on a Fréchet space $X$ is called a hypercyclic operator if it admits a hypercyclic vector $x \in X$, that is, if there is a vector $x \in X$ such that the orbit $\left\{x, T x, \cdots, T^{n} x, \cdots\right\}$ is dense in $X$, where $T^{n}$ denotes the $n$-th iterate of $T$. Hypercyclic operators have been studied by many authors; we refer to $2,3,5,7,9,11,12,16,17,18$ for some recent works.

Throughout, let $G$ be a locally compact group with identity $e$ and a right Haar measure $\lambda$. Since a Fréchet space admits a hypercyclic operator if, and only if, it is separable and infinite-dimensional, the question of hypercyclicity is meaningful for the complex Lebesgue space $L^{p}(G)$, with respect to $\lambda$, only when $G$ is second countable and $1 \leq p<\infty$ which will be assumed henceforth. Given a compact subgroup $H$ of $G$, the right coset space $G / H$ admits a $G$-invariant measure $\nu=$ $\lambda \circ q^{-1}$, where $q: G \longrightarrow G / H$ is the quotient map. Convolution operators on the Lebesgue space $L^{p}(G / H)$ with respect to $\nu$ can be lifted naturally to $L^{p}(G)$ (cf. [8, p. 77]). Hence all hypercyclic results in this paper for $L^{p}(G)$ can be extended without difficulty to $L^{p}(G / H)$, as in [6]. For simplicity and to expose the essential ideas, we will confine our discussion to $L^{p}(G)$.

Received by the editors October 7, 2009 and, in revised form, July 28, 2010.

2010 Mathematics Subject Classification. Primary 47A16, 47B37, 47B38, 44A35, 43A15.

Key words and phrases. Hypercyclic operator, topologically mixing operator, convolution, locally compact group, $L^{p}$-space.

(C)2011 American Mathematical Society Reverts to public domain 28 years from publication 
A bounded continuous function $w: G \rightarrow(0, \infty)$ is called a weight on $G$. Let $a \in G$ and let $\delta_{a}$ be the unit point mass at $a$. A weighted translation on $G$ is a weighted convolution operator $T_{a, w}: L^{p}(G) \longrightarrow L^{p}(G)$ defined by

$$
T_{a, w}(f):=w T_{a}(f) \quad\left(f \in L^{p}(G)\right),
$$

where $w$ is a weight on $G$ and $T_{a}(f)=f * \delta_{a} \in L^{p}(G)$ is the convolution

$$
\left(f * \delta_{a}\right)(x):=\int_{G} f\left(x y^{-1}\right) d \delta_{a}(y)=f\left(x a^{-1}\right) \quad(x \in G),
$$

which is just the right translation of $f$ by $a^{-1}$.

A translation operator $T_{a}$ is never hypercyclic since $\left\|T_{a}\right\| \leq 1$. However, a weighted translation operator $w T_{a}=T_{a, w}$ can be hypercyclic. Our objective is to address the natural question of hypercyclicity of $T_{a, w}$. For discrete groups $G$, we answer the question completely by giving a necessary and sufficient condition in terms of the weight $w$ for $T_{a, w}$ to be hypercyclic. For non-discrete groups, the same condition also characterizes hypercyclicity of $T_{a, w}$ when the element $a$ is aperiodic.

Before discussing the details, we first observe that certain weights and elements in $G$ can be excluded from our consideration. Plainly, if $\|w\|_{\infty} \leq 1$, then $\left\|T_{a, w}\right\| \leq 1$ and $T_{a, w}$ is never hypercyclic. The following lemma reveals that we can ignore torsion elements in $G$, a fact not noted in [6].

An element $a$ in a group $G$ is called a torsion element if it is of finite order. In a locally compact group $G$, an element $a \in G$ is called periodic 13] (or compact 14, $9.9]$ ) if the closed subgroup $G(a)$ generated by $a$ is compact. We call an element in $G$ aperiodic if it is not periodic. For discrete groups, periodic and torsion elements are identical; in other words, aperiodic elements are the non-torsion elements.

Lemma 1.1. Let $G$ be a locally compact group and let $a \in G$ be a torsion element. Then any weighted translation $T_{a, w}: L^{p}(G) \longrightarrow L^{p}(G)$ is not hypercyclic, for $1 \leq p<\infty$.

Proof. Let $a$ have order $d$ say, and consider the product $\omega_{d}$ of the first $d$ translations of the weight $w$ :

$$
\omega_{d}(x)=w(x) w\left(x a^{-1}\right) \cdots w\left(x a^{-(d-1)}\right) \quad(x \in G) .
$$

Either $\left\|\omega_{d}\right\|_{\infty} \leq 1$ or $\left\|\omega_{d}\right\|_{\infty}>1$. We show in both cases that $T_{a, w}$ is not hypercyclic.

In the first case, we make use of $a^{d}=e$ and deduce

$$
T_{a, w}^{n}=\omega_{d}^{\left[\frac{n}{d}\right]} T_{a, w}^{j} \quad(n \in \mathbb{N}),
$$

where $n=d\left[\frac{n}{d}\right]+j$ with $j \in\{0,1, \cdots, d-1\}$ and $T_{a, w}^{0}$ is the identity map. For each $f \in L^{p}(G)$, the orbit of $f$ is given by

$$
\left\{f, \omega_{d} f, \omega_{d}^{2} f, \cdots\right\} \cup\left\{f_{1}, \omega_{d} f_{1}, \omega_{d}^{2} f_{1}, \cdots\right\} \cup \cdots \cup\left\{f_{d-1}, \omega_{d} f_{d-1}, \omega_{d}^{2} f_{d-1}, \cdots\right\},
$$

where the function $f_{j}$ denotes the $j$-th iterate $T_{a, w}^{j} f$, for $j \in\{1,2, \cdots, d-1\}$. Since $\left\|\omega_{d}\right\|_{\infty} \leq 1$, the orbit of $f$ is bounded and hence cannot be dense in $L^{p}(G)$. Therefore $T_{a, w}$ does not admit a hypercyclic vector in this case.

Next, suppose $T_{a, w}$ is hypercyclic for the case $\left\|\omega_{d}\right\|_{\infty}>1$. We deduce a contradiction. By continuity of $w$ and regularity of $\lambda$, there is a compact set $U \subset G$ such that $\lambda(U)>2 \varepsilon^{p}$ for some $\varepsilon>0$ and $\omega_{d}(x)>1$ for all $x \in U$. We note that the $d$-th interate $T_{a, w}^{d}$ is also hypercyclic by [1, Theorem 1]. 
Let $\chi_{U}$ be the characteristic function of $U$. Then $\chi_{U} \in L^{p}(G)$. Since the hypercyclic vectors for $T_{a, w}^{d}$ are dense, there exist a hypercyclic vector $f \in L^{p}(G)$ and sufficiently large $n \in \mathbb{N}$ such that

$$
\left\|f-2 \chi_{U}\right\|_{p}<\varepsilon \text { and }\left\|\left(T_{a, w}^{d}\right)^{n} f\right\|_{p}<\varepsilon .
$$

Let $V=\{x \in U:|f(x)|<1\}$. Then

$$
\varepsilon^{p}>\left\|f-2 \chi_{U}\right\|_{p}^{p} \geq \int_{V}|f(x)-2|^{p} d \lambda(x) \geq \lambda(V) .
$$

Let $W=U \backslash V$. Then we have

$$
\begin{aligned}
\varepsilon^{p} & >\left\|\left(T_{a, w}^{d}\right)^{n} f\right\|_{p}^{p} \\
& \geq \int_{W}\left|\omega_{d}^{n}(x) f(x)\right|^{p} d \lambda(x) \geq \lambda(W) .
\end{aligned}
$$

Hence $\lambda(U)=\lambda(V)+\lambda(W)<2 \varepsilon^{p}$, which is a contradiction. Therefore $T_{a, w}$ is not hypercyclic in the second case either.

Remark 1.2. In the case of $\left\|\omega_{d}\right\|_{\infty} \leq 1$ above, one can also apply [1, Theorem 1] to deduce that $T_{a, w}$ is not hypercyclic by observing $T_{a, w}^{d}=T_{e, w_{d}}$.

\section{Characterization of hypercyclicity}

We established in 6] a criterion of hypercyclicity for a weighted translation $T_{a, w}$ by an aperiodic element $a$ in a discrete group $G$. We extend this result to non-discrete groups in this section. It has been shown in [6, Lemma 2.7] that an element $a$ in a discrete group $G$ is aperiodic if, and only if, for any finite subset $K \subset G$, there exists $N \in \mathbb{N}$ such that $K \cap K a^{n}=\emptyset$ (equivalently, $K \cap K a^{-n}=\emptyset$ ) for $n>N$. It is the latter condition which was used to deduce the necessary weight condition for hypercyclicity. We first extend the above criterion of aperiodicity to non-discrete groups.

Lemma 2.1. An element a in a second countable group $G$ is aperiodic if, and only if, for each compact subset $K \subset G$, there exists $N \in \mathbb{N}$ such that $K \cap K a^{n}=\emptyset$ for $n>N$.

Proof. Let $a$ be aperiodic. Then the closed subgroup $G(a)$ generated by $a$ in $G$ is not compact. By [14, Theorem 5.14], the element $a$ is contained in a compactly generated closed subgroup of $G(a)$. It follows that $G(a)$ is a compactly generated abelian group and is therefore topologically isomorphic to a direct product $\mathbb{R}^{n} \times$ $\mathbb{Z}^{m} \times F$ where $F$ is a compact group, by [14, Theorem 9.8]. Moreover, $a$ identifies with an element in $\left(\mathbb{R}^{n} \times \mathbb{Z}^{m} \times F\right) \backslash(\{0\} \times\{0\} \times F)$ since $G(a)$ is not compact. Hence for any sequence $\left(n_{k}\right)$ in $\mathbb{N}$, the sequence $\left(a^{n_{k}}\right)$ in $G$ cannot converge to the identity $e$. Now let $K$ be any non-empty compact subset of $G$. If $K \cap K a^{n} \neq \emptyset$ frequently for $n$, then the sequence $\left(a^{n}\right)$ lies in the compact set $K^{-1} K$ frequently, and we can find a subsequence $\left(a^{n_{k}}\right)$ of $\left(a^{n}\right)$ converging to some $b \in K^{-1} K$. Hence the sequence $\left(a^{n_{k+1}-n_{k}}\right)$ converges to $e$, which is impossible. This proves $K \cap K a^{n}=\emptyset$ from some $n$ onwards.

Conversely, let $a$ be periodic. By compactness of $G(a)$, we can find a subsequence $\left(a^{n_{k}}\right)$ of $\left(a^{n}\right)$ converging to some $b \in G(a)$, and hence $a^{n_{k+1}-n_{k}} \rightarrow e$. Therefore the compact set $K=\{e\} \cup\left\{a^{n_{k+1}-n_{k}}: k=1,2, \cdots\right\}$ satisfies $K \cap K a^{n_{k+1}-n_{k}} \neq \emptyset$ for each $k$. 
Remark 2.2. The above proof also shows that an element $a$ in a second countable group is periodic if, and only if, there is a sequence $\left(n_{k}\right)$ in $\mathbb{N}$ such that $\lim _{k \rightarrow \infty} a^{n_{k}}=e$. In many familiar non-discrete groups, including the additive group $\mathbb{R}^{n}$, the Heisenberg group and the affine group, all elements except the identity are aperiodic. It should be noted, however, that non-torsion elements in a non-discrete group need not be aperiodic. For instance, the circle group $\mathbb{T}$ is compact and hence all elements in it are periodic, but an element $e^{i \theta}$ is non-torsion if $\frac{\theta}{2 \pi}$ is irrational.

We are now ready to show how hypercyclicity of the weighted translation $T_{a, w}$ depends on the behavior of successive translations of $w$ by $a$. We will make use of the equivalence of hypercyclicity and topological transitivity [11; namely, an operator $T$ on a Banach space $X$ is hypercyclic if and only if for any non-empty open sets $U$ and $V$ in $X$, we have $T^{n}(U) \cap V \neq \emptyset$ for some $n \in \mathbb{N}$. If $T$ satisfies the stronger condition that $T^{n}(U) \cap V \neq \emptyset$ from some $n$ onwards, then $T$ is said to be topologically mixing.

Theorem 2.3. Let $G$ be a locally compact group and let a be an aperiodic element in $G$. Let $1 \leq p<\infty$ and $T_{a, w}$ be a weighted translation on $L^{p}(G)$. The following conditions are equivalent:

(i) $T_{a, w}$ is hypercyclic.

(ii) For each compact subset $K \subset G$ with $\lambda(K)>0$, there is a sequence of Borel sets $\left(E_{k}\right)$ in $K$ such that $\lambda(K)=\lim _{k \rightarrow \infty} \lambda\left(E_{k}\right)$ and both sequences

$$
w_{n}:=\prod_{s=1}^{n} w * \delta_{a^{-1}}^{s} \quad \text { and } \quad \widetilde{w}_{n}:=\left(\prod_{s=0}^{n-1} w * \delta_{a}^{s}\right)^{-1}
$$

admit respectively subsequences $\left(w_{n_{k}}\right)$ and $\left(\widetilde{w}_{n_{k}}\right)$ satisfying

$$
\lim _{k \rightarrow \infty}\left\|\left.w_{n_{k}}\right|_{E_{k}}\right\|_{\infty}=\lim _{k \rightarrow \infty}\left\|\left.\widetilde{w}_{n_{k}}\right|_{E_{k}}\right\|_{\infty}=0 .
$$

Proof. (i) $\Rightarrow$ (ii). We extend the proof of [19, Theorem 2.1] to our setting. Let $T_{a, w}$ be hypercyclic. Let $K \subset G$ be a compact set with $\lambda(K)>0$. Let $\varepsilon>0$. By aperiodicity of $a$, there is some $N$ such that $K \cap K a^{-n}=\emptyset$ for $n>N$. Let $\chi_{K} \in L^{p}(G)$ be the characteristic function of $K$. Choose $0<\delta<\frac{\varepsilon}{1+\varepsilon}$. By density of the hypercyclic vectors for $T_{a, w}$, there exist a hypercyclic vector $f \in L^{p}(G)$ and some $m>N$ such that

$$
\left\|f-\chi_{K}\right\|_{p}<\delta^{2} \quad \text { and } \quad\left\|T_{a, w}^{m} f-\chi_{K}\right\|_{p}<\delta^{2} .
$$

Let $A_{\delta}=\{x \in K:|f(x)-1| \geq \delta\}$. Then we have

$$
|f(x)|>1-\delta \quad\left(x \in K \backslash A_{\delta}\right)
$$

and $\lambda\left(A_{\delta}\right)<\delta^{p}$. Indeed, either $\lambda\left(A_{\delta}\right)=0$ or

$$
\begin{aligned}
\delta^{2 p}>\left\|f-\chi_{K}\right\|_{p}^{p} & =\int_{G}\left|f(x)-\chi_{K}(x)\right|^{p} d \lambda(x) \\
& \geq \int_{K}|f(x)-1|^{p} d \lambda(x) \\
& \geq \int_{A_{\delta}}|f(x)-1|^{p} d \lambda(x) \geq \delta^{p} \lambda\left(A_{\delta}\right),
\end{aligned}
$$

giving $\lambda\left(A_{\delta}\right)<\delta^{p}$. 
Let $B_{\delta}=\{x \in G \backslash K:|f(x)| \geq \delta\}$. Then we have

$$
|f(x)|<\delta \quad \text { for } \quad x \in(G \backslash K) \backslash B_{\delta}
$$

and as before, $0 \leq \lambda\left(B_{\delta}\right)<\delta^{p}$ since

$$
\delta^{2 p}>\int_{G \backslash K}\left|f(x)-\chi_{K}(x)\right|^{p} d \lambda(x) \geq \int_{B_{\delta}}|f(x)|^{p} d \lambda(x) \geq \delta^{p} \lambda\left(B_{\delta}\right) .
$$

Let $C_{m, \delta}=\left\{x \in K:\left|\widetilde{w}_{m}(x)^{-1} f\left(x a^{-m}\right)-1\right| \geq \delta\right\}$. Then we have

$$
\widetilde{w}_{m}(x)^{-1}\left|f\left(x a^{-m}\right)\right|>1-\delta \quad\left(x \in K \backslash C_{m, \delta}\right)
$$

and also

$$
\begin{aligned}
\delta^{2 p} & >\left\|T_{a, w}^{m} f-\chi_{K}\right\|_{p}^{p}=\int_{G}\left|T_{a, w}^{m} f(x)-\chi_{K}(x)\right|^{p} d \lambda(x) \\
& \geq \int_{C_{m, \delta}}\left|w(x) w\left(x a^{-1}\right) \cdots w\left(x a^{-(m-1)}\right) f\left(x a^{-m}\right)-1\right|^{p} d \lambda(x) \geq \delta^{p} \lambda\left(C_{m, \delta}\right)
\end{aligned}
$$

which implies $\lambda\left(C_{m, \delta}\right)<\delta^{p}$. Since $K \cap K a^{-m}=\emptyset$, we have

$$
\widetilde{w}_{m}(x)<\frac{\left|f\left(x a^{-m}\right)\right|}{1-\delta}<\frac{\delta}{1-\delta}<\varepsilon \quad \text { for } \quad x \in K \backslash\left(C_{m, \delta} \cup B_{\delta} a^{m}\right) .
$$

Let $D_{m, \delta}=\left\{x \in K:\left|w_{m}(x) f(x)\right| \geq \delta\right\}$. Then we have

$$
w_{m}(x)|f(x)|<\delta \quad\left(x \in K \backslash D_{m, \delta}\right),
$$

and from $K \cap K a^{m}=\emptyset$ we deduce

$$
\begin{aligned}
\delta^{2 p} & >\int_{G}\left|w(x) w\left(x a^{-1}\right) \cdots w\left(x a^{-(m-1)}\right) f\left(x a^{-m}\right)-\chi_{K}(x)\right|^{p} d \lambda(x) \\
& =\int_{G}\left|w\left(x a^{m}\right) w\left(x a^{m-1}\right) \cdots w(x a) f(x)-\chi_{K}\left(x a^{m}\right)\right|^{p} d \lambda(x) \\
& \geq \int_{D_{m, \delta}}\left|w\left(x a^{m}\right) w\left(x a^{m-1}\right) \cdots w(x a) f(x)\right|^{p} d \lambda(x) \geq \delta^{p} \lambda\left(D_{m, \delta}\right)
\end{aligned}
$$

which implies $\lambda\left(D_{m, \delta}\right)<\delta^{p}$. It follows that

$$
w_{m}(x)<\frac{\delta}{|f(x)|}<\frac{\delta}{1-\delta}<\varepsilon \quad \text { for } \quad x \in K \backslash\left(D_{m, \delta} \cup A_{\delta}\right) .
$$

Let $E_{m, \delta}=K \backslash\left(A_{\delta} \cup B_{\delta} a^{m} \cup C_{m, \delta} \cup D_{m, \delta}\right)$. Then we have $\lambda\left(K \backslash E_{m, \delta}\right)<4 \delta^{p}$, $\left\|\left.w_{m}\right|_{E_{m, \delta}}\right\|_{\infty}<\varepsilon$ and $\left\|\left.\widetilde{w}_{m}\right|_{E_{m, \delta}}\right\|_{\infty}<\varepsilon$.

For $k=1,2, \cdots$, the above arguments enable us to construct a Borel set $E_{k} \subset K$ and inductively $n_{1}<\cdots<n_{k}<\cdots$ such that $\lambda\left(K \backslash E_{k}\right)<4\left(\frac{1}{k}\right)^{p},\left\|\left.w_{n_{k}}\right|_{E_{k}}\right\|_{\infty}<\frac{1}{k}$ and $\left\|\left.\widetilde{w}_{n_{k}}\right|_{E_{k}}\right\|_{\infty}<\frac{1}{k}$. Therefore the sequence $\left(E_{k}\right)$ satisfies condition (ii).

(ii) $\Rightarrow$ (i). We show that $T_{a, w}$ is topologically transitive. Let $U$ and $V$ be nonempty open subsets of $L^{p}(G)$. Since the space $C_{c}(G)$ of continuous functions on $G$ with compact support is dense in $L^{p}(G)$, we can pick $f, g \in C_{c}(G)$ with $f \in U$ and $g \in V$. Let $K$ be the union of the compact supports of $f$ and $g$. Let $E_{k} \subset K$ and the subsequences $\left(w_{n_{k}}\right)$ and $\left(\widetilde{w}_{n_{k}}\right)$ satisfy condition (ii).

By aperiodicity of $a$, there exists $M \in \mathbb{N}$ such that $K \cap K a^{ \pm n}=\emptyset$ for all $n>M$. 
First, we show that $\left\|T_{a, w}^{n_{k}}\left(f \chi_{E_{k}}\right)\right\|_{p} \rightarrow 0$ as $k \rightarrow \infty$. Let $\varepsilon>0$. There exists $N \in \mathbb{N}$ such that $n_{k}>M$ and $w_{n_{k}}^{p}<\frac{\varepsilon}{\|f\|_{p}^{p}}$ on $E_{k}$ for $k>N$. Hence

$$
\begin{aligned}
\left\|T_{a, w}^{n_{k}}\left(f \chi_{E_{k}}\right)\right\|_{p}^{p} & =\int_{E_{k} a^{n_{k}}}\left|w(x) w\left(x a^{-1}\right) \cdots w\left(x a^{-\left(n_{k}-1\right)}\right)\right|^{p}\left|f\left(x a^{-n_{k}}\right)\right|^{p} d \lambda(x) \\
& =\int_{E_{k}}\left|w\left(x a^{n_{k}}\right) w\left(x a^{n_{k}-1}\right) \cdots w(x a)\right|^{p}|f(x)|^{p} d \lambda(x) \\
& =\int_{E_{k}} w_{n_{k}}^{p}(x)|f(x)|^{p} d \lambda(x)<\varepsilon
\end{aligned}
$$

for $k>N$.

We define a self-map $S_{a, w}$ on the subspace $L_{c}^{p}(G)$ of functions in $L^{p}(G)$ with compact support by

$$
S_{a, w}(h)=\frac{h}{w} * \delta_{a^{-1}} \quad\left(h \in L_{c}^{p}(G)\right)
$$

so that

$$
T_{a, w} S_{a, w}(h)=h \quad\left(h \in L_{c}^{p}(G)\right) .
$$

Applying similar arguments to the iterates $S_{a, w}^{n_{k}}$, using the sequence $\left(\widetilde{w}_{n_{k}}\right)$, yields

$$
\begin{aligned}
\lim _{k \rightarrow \infty} & \left\|S_{a, w}^{n_{k}}\left(g \chi_{E_{k}}\right)\right\|_{p}^{p} \\
& =\lim _{k \rightarrow \infty} \int_{E_{k} a^{-n_{k}}} \frac{1}{\left|w(x a) w\left(x a^{2}\right) \cdots w\left(x a^{n_{k}}\right)\right|^{p}}\left|g\left(x a^{n_{k}}\right)\right|^{p} d \lambda(x)=0 .
\end{aligned}
$$

For each $k \in \mathbb{N}$, we let

$$
v_{k}=f \chi_{E_{k}}+S_{a, w}^{n_{k}}\left(g \chi_{E_{k}}\right) \in L^{p}(G) .
$$

Then

and

$$
\left\|v_{k}-f\right\|_{p}^{p} \leq\|f\|_{\infty}^{p} \lambda\left(K \backslash E_{k}\right)+\left\|S_{a, w}^{n_{k}}\left(g \chi_{E_{k}}\right)\right\|_{p}^{p}
$$

$$
\left\|T_{a, w}^{n_{k}} v_{k}-g\right\|_{p}^{p} \leq\left\|T_{a, w}^{n_{k}}\left(f \chi_{E_{k}}\right)\right\|_{p}^{p}+\|g\|_{\infty}^{p} \lambda\left(K \backslash E_{k}\right) .
$$

Hence $\lim _{k \rightarrow \infty} v_{k}=f$ and $\lim _{k \rightarrow \infty} T_{a, w}^{n_{k}} v_{k}=g$ which implies $T_{a, w}^{n_{k}}(U) \cap V \neq \emptyset$ for some $k$.

Remark 2.4. One can construct examples of weights $w$, as in [6. Example 2.5], satisfying condition (ii) above. This condition can be seen to be weaker than the sufficient condition for hypercyclicity in [6, Proposition 2.2], via Egoroff's theorem. If $G$ is a discrete group, condition (ii) is equivalent to the fact that both sequences $\left(w_{n}\right)$ and $\left(\widetilde{w}_{n}\right)$ admit subsequences $\left(w_{n_{k}}\right)$ and $\left(\widetilde{w}_{n_{k}}\right)$ which converge to 0 pointwise in $G$. Hence Theorem 2.3 is identical to [6, Theorem 2.8] for discrete groups. It also implies that if $T_{a, w}: L^{p}(G) \longrightarrow L^{p}(G)$ is hypercyclic for some $p \in[1, \infty)$, then it is so for all $p \in[1, \infty)$.

It has been shown in 4 that an operator $T$ satisfies the Hypercyclic Criterion if, and only if, the direct sum $T \oplus T$ is hypercyclic. Condition (ii) in Theorem 2.3 also implies that $T_{a, w} \oplus T_{a, w}$ is topologically transitive. Indeed, if one considers four non-empty open sets $U_{j}$ and $V_{j}(\mathrm{j}=1,2)$ in $L^{p}(G)$ and pick $f_{j}, g_{j} \in C_{c}(G)$ with $f_{j} \in U_{j}$ and $g_{j} \in V_{j}$, then the same arguments in the proof of (ii) $\Rightarrow$ (i) in Theorem 2.3 can be applied to these functions to obtain two sequences $\left(v_{1, k}\right)$ and $\left(v_{2, k}\right)$ in $L^{p}(G)$ satisfying $\lim _{k \rightarrow \infty} v_{j, k}=f_{j}$ and $\lim _{k \rightarrow \infty} T_{a, w}^{n_{k}} v_{j, k}=g_{j}$ for $j=1,2$, yielding $T_{a, w}^{n_{k}}\left(U_{j}\right) \cap V_{j} \neq \emptyset$ for some $k$. Hence we can draw the following conclusion. 
Corollary 2.5. Let $G$ be a locally compact group and let a be an aperiodic element in $G$. Let $1 \leq p<\infty$ and $T_{a, w}$ be a weighted translation on $L^{p}(G)$. The following conditions are equivalent:

(i) $T_{a, w}$ satisfies the Hypercyclic Criterion.

(ii) $T_{a, w}$ is hypercyclic.

(iii) $T_{a, w} \oplus T_{a, w}$ is hypercyclic.

(iv) $T_{a, w}$ is weakly mixing.

Using similar arguments as in the proof of Theorem 2.3, we can also characterize topological mixing weighted translations for non-discrete groups.

Corollary 2.6. Let $a$ be an aperiodic element in $G$ and let $T_{a, w}$ be a weighted translation on $L^{p}(G)$ for $1 \leq p<\infty$. The following conditions are equivalent:

(i) $T_{a, w}$ is topologically mixing.

(ii) For every compact subset $K$ of $G$ with $\lambda(K)>0$, there is a sequence of Borel sets $\left(E_{n}\right)$ in $K$ such that $\lambda(K)=\lim _{n \rightarrow \infty} \lambda\left(E_{n}\right)$ and both sequences

$$
w_{n}:=\prod_{s=1}^{n} w * \delta_{a^{-1}}^{s} \quad \text { and } \quad \widetilde{w}_{n}:=\left(\prod_{s=0}^{n-1} w * \delta_{a}^{s}\right)^{-1}
$$

satisfy

$$
\lim _{n \rightarrow \infty}\left\|\left.w_{n}\right|_{E_{n}}\right\|_{\infty}=\lim _{n \rightarrow \infty}\left\|\left.\widetilde{w}_{n}\right|_{E_{n}}\right\|_{\infty}=0
$$

Proof. The proof of (ii) $\Rightarrow$ (i) is identical to that in Theorem 2.3, using the full sequences $\left(w_{n}\right)$ and $\left(\widetilde{w}_{n}\right)$ instead of subsequences. For the converse, let $K$ be a compact set in $G$ with $\lambda(K)>0$. Let $\varepsilon>0$ and $\chi_{K} \in L^{p}(G)$ be the characteristic function as defined in the proof of Theorem 2.3. Choose $0<\delta<\frac{\varepsilon}{1+\varepsilon}$ and let $U=\left\{f \in L^{p}(G):\left\|f-\chi_{K}\right\|_{p}<\delta^{2}\right\}$. By the topologically mixing assumption, there exists $N \in \mathbb{N}$ such that $n>N$ implies $K \cap K a^{-n}=\emptyset$ and

$$
T_{a, w}^{n}(U) \cap U \neq \emptyset .
$$

We can therefore pick, for each $n>N$, a function $f_{n} \in U$ with $T_{a, w}^{n} f_{n} \in U$ which gives

$$
\left\|f_{n}-\chi_{K}\right\|_{p}<\delta^{2} \quad \text { and } \quad\left\|T_{a, w}^{n} f_{n}-\chi_{K}\right\|_{p}<\delta^{2} .
$$

Using this for each $f_{n}$ and repeating the arguments in the proof of Theorem 2.3. we find Borel sets $E_{n} \subset K$ with $\lambda(K)=\lim _{n \rightarrow \infty} \lambda\left(E_{n}\right)$ such that $\left\|\left.w_{n}\right|_{E_{n}}\right\|_{\infty}<\frac{1}{n}$ and $\left\|\left.\widetilde{w}_{n}\right|_{E_{n}}\right\|_{\infty}<\frac{1}{n}$, proving condition (ii).

\section{REFERENCES}

1. S. I. Ansari, Hypercyclic and cyclic vectors, J. Funct. Anal. 128 (1995) 374-383. MR.1319961 (96h:47002)

2. F. Bayart and É. Matheron, Hypercyclic operators failing the hypercyclicity criterion on classical Banach spaces, J. Funct. Anal. 250 (2007) 426-441. MR2352487 (2008k:47016)

3. L. Bernal-González and K.-G. Grosse-Erdmann, The hypercyclicity criterion for sequences of operators, Studia Math. 157 (2003) 17-32. MR1980114 (2003m:47013)

4. J. Bès and A. Peris, Hereditarily hypercyclic operators, J. Funct. Anal. 167 (1999) 94-112. MR:1710637 (2000f:47012)

5. J. Bonet, Hypercyclic and chaotic convolution operators, J. London Math. Soc. 62 (2000) 253-262. MR 1772185 (2001g:47053)

6. C. Chen and C-H. Chu, Hypercyclicity of weighted convolution operators on homogeneous spaces, Proc. Amer. Math. Soc. 137 (2009) 2709-2718. MR2497483 
7. J-C. Chen and S-Y. Shaw, Topological mixing and hypercyclicity criterion for sequences of operators, Proc. Amer. Math. Soc. 134 (2006) 3171-3179. MR2231900 (2007d:47008)

8. C-H. Chu, Matrix convolution operators on groups, Lecture Notes in Math. 1956, SpringerVerlag, Heidelberg, 2008. MR2450997 (2010h:43009)

9. G. Costakis and M. Sambarino, Topologically mixing hypercyclic operators, Proc. Amer. Math. Soc. 132 (2004) 385-389. MR2022360 (2004i:47017)

10. R.M. Gethner and J.H. Shapiro, Universal vectors for operators on spaces of holomorphic functions, Proc. Amer. Math. Soc. 100 (1987) 281-288. MR.884467(88g:47060)

11. K.-G. Grosse-Erdmann, Universal families and hypercyclic operators, Bull. Amer. Math. Soc. (N.S.) 36 (1999) 345-381. MR.1685272(2000c:47001)

12. K.-G. Grosse-Erdmann, Recent developments in hypercyclicity, RACSAM Rev. R. Acad. Cien. Ser. A. Mat. 97 (2003) 273-286. MR2068180 (2005c:47010)

13. S. Grosser and M. Moskowitz, On central topological groups, Trans. Amer. Math. Soc. 127 (1967) 317-340. MR0209394 (35:292)

14. E. Hewitt and K.A. Ross, Abstract harmonic analysis, Springer-Verlag, Heidelberg, 1979. MR.551496 (81k:43001)

15. C. Kitai, Invariant closed sets for linear operators, PhD Thesis, University of Toronto, 1982.

16. F. León-Saavedra and V. Müller, Rotations of hypercyclic and supercyclic operators, Integr. Equat. Oper. Th. 50 (2004) 385-391. MR2104261 (2005g:47009)

17. A. Peris and L. Saldivia, Syndetically hypercyclic operators, Integr. Equat. Oper. Th. 51 (2005) 275-281. MR.2120081 (2005h:47017)

18. H. Petersson, Spaces that admit hypercyclic operators with hypercyclic adjoints, Proc. Amer. Math. Soc. 134 (2006) 1671-1676. MR2204278 (2007b:47020)

19. H. Salas, Hypercyclic weighted shifts, Trans. Amer. Math. Soc. 347 (1995) 993-1004. MR.1249890 (95e:47042)

School of Mathematical Sciences, Queen Mary, University of London, Mile End Road, London E1 4NS, United Kingdom

E-mail address: c.chen@qmul.ac.uk

School of Mathematical Sciences, Queen Mary, University of London, Mile End RoAd, London E1 4NS, United Kingdom

E-mail address: c.chu@qmul.ac.uk 